\title{
BREVE RESEÑ A DE LA ENFERMERÍA DOMICILIARIA: UNA PERSPECTIVA DE LA ATENCIÓN PRIVADA
}

\author{
BRIEF OUTLOOK OF DOMICILIARY NURSING: A PERSPECTIVE \\ OF PRIVATE CARE
}

\author{
ANGÉLICA ORB* y ALICIA SANTIAGOS**
}

\begin{abstract}
RESUMEN
La atención domiciliaria prestada a un sector de la comunidad santiaguina es descrita en esteartículo. La selección de pacientes, la participación de la familia, los costos del servicio y cargas de trabajo de las enfermeras son discutidos como también las dificultades del cuidado en el domicilio. Esteartículo examina un sistema deatención domiciliaria de tipo privado que usualmente no es promovida o conocida en el ambiente chileno. El artículo recomienda una revisión sistemática del cuidado de enfermería domiciliaria y señala que las pautas de este modelo son las bases del cuidado moderno de los programas de atención en el hogar.
\end{abstract}

Palabras claves: Cuidado domiciliario, cuidado en el hogar, salud domiciliaria.

\begin{abstract}
An insight into the domiciliary nursing care provided to a section of Santiago's community is presented. Patient selection, family participation, as well as the cost of the service offered and nurses worked load are discussed. Some of the difficulties encountered in the provision of care are also highlighted. The article provides a close examination into a private domiciliary care system that usually is not reported. The paper finishes recommending a systematic review of domiciliary nursing as well as highlighting the guiding principles of this model of care which are the bases of the modern approach to the home care programs.
\end{abstract}

Keywords: Domiciliary care, home care, home health care.

Recepción: 01.02.2004. Aceptación: 15.04.2005

La atención de enfermería hospitalaria está cambiando. El enfermo agudo está siendo atendido en su domicilio. A pesar queestemodelo deatención no es nuevo, se está volviendo a lo que en el periodo pre industrial era la forma normal de cuidado (Arno, Bonuck \& Padgug, cita en Duke \& Street, 2003). H oy en día, las tendencias de salud son las de mantener al paciente el tiempo más corto posible en el hospital y continuar con su cuidado en el hogar. La tendencia es ofrecer servicios de salud comunitaria, que puedan disminuir los costos de salud (Kearney, York \& Deatrick, 2000). El modelo deatención domiciliariadel enfermo agudo son las bases del cuidado moderno de los programas de atención en el hogar y una de las alternativas (Joel, 2001) más deseables. Los enfermos permanecen en su ambientepropio, no están expuestosa las posibles infecciones y no tienen que adaptarsea la hospitalización (Duke \& Street, 2003). El hogar y la familia son elementos de gran importancia en la atención domiciliaria, los que juegan un rol preponderanteen el bienestar y

\footnotetext{
*RN , PhD, M ACE, Alpha Chi Senior Lecturer, Escuela de Enfermería y Obstetricia, Curtin University Of Technology, Perth, Western Australia. Correspondencia a: Dra. A. Orb, GPO Box U 1987, Perth, Western Australia 6845. Fax (08) 9266 2959. E-mail: A. Orb@.curtin.edu.au

**Enfermera Clínica, Home M edical Clinic Las Condes, Santiago, Chile.
} 
recuperación del paciente (Wright \& Leahey, 1994). Por otro lado, las enfermeras también reconocen la importancia e influencia de la familia cuando se pierde la salud (Wright \& Leahey, 1994). Por ejemplo, Kellett (1999) en su estudio sobre la familia encontró que el hecho de hacer participar a ésta en el cuidado del enfermo tieneun significado degran magnitud para el paciente. Otros, como Joel (2001), reconocen la satisfacción que las enfermeras han expresado en relación con el alto grado de autonomía profesional en el cuidado en el hogar. Esteambientebajo ciertas circunstanciasesel mejor lugar dondeel paciente puedemantener el control desu cuidado. Para más claridad, el concepto de cliente o paciente se usará indistintamente en este artículo. También es importante destacar quela literatura en esta área usa los términos'enfermería domiciliaria' (domiciliary nursing) y 'cuidado en el hogar' (home care) sin hacer mayor distinción.

En Chile desde el año 1989 existela atención domiciliaria a nivel privado. ADP fue la primera empresa que trasladó la atención domiciliaria privada al ambientedel hogar. En otras palabras, llevó al hogar el cuidado que generalmente se prestaba en el hospital, adaptando estemodelo deatención a las necesidades de los enfermos. La atención domiciliaria no solamentees facilitada por la presencia del equipo de salud, sino también a través del traslado del material y equipos necesarios para alcanzar una óptima atención de enfermería.

Es importante enfatizar que los beneficiarios de este sistema son clientes que tienen seguros de salud privado o que, sin tener aqueIlos seguros, pueden financiar los gastos personalmente. Los servicios domiciliarios otorgan atención médica, de enfermería, de laboratorio clínico y defisioterapia, para mencionar algunos. Además el equipo desalud cuenta con un personal capacitado y con la experiencia para entregar estetipo deatención. El servicio de enfermería tiene una dotación de enfermeras, auxiliares de enfermería y perso- nal deservicio queprestan atención continuada por 24 horas al día. Desgraciadamente, poco se sabe de la atención domiciliaria prestada por este grupo de enfermeras en Santiago deChile. Unarevisión dela literaturamostró que la mayoría de la información relacionada a la atención domiciliaria proviene de los Estados Unidos de Norte América (Flynn \& Deatrick, 2003; Hughes, Hodgson, Muller et al., 2000; Kearney, York \& Deatrick, 2000). En cambio, en la literatura británica el término visita domiciliaria (health visiting) se usa para referirse a la prevención de la salud en el hogar (Bryans, 2004). El objetivo de esteartículo es describir la atención de enfermería prestada a los usuarios de este sistema de salud que viven en la cuidad de Santiago de Chile. La mayor parte de la información presentada en esteartículo es detipo anecdótico, emanada de las conversaciones y visitas domiciliarias con la segunda autora.

\section{UBICACIÓN GEOGRÁFICA DEL SERVICIO}

Chile tiene una población cercana a los 15 millones de habitantes (INE, 2002), los cualesviven entre las latitudes sur del paralelo 30 y 42 (Schneider, 2001). A proximadamente cinco millones viven en la capital, Santiago (INE, 2002), que tiene un total de 32 comunas, distribuidas en zona, norte, sur, occidente y oriente. El servicio descrito en este artículo tiene un promedio de atención de 50 pacientes diarios, que se distribuyen principalmente en el área oriente con una población de aproximadamente 800.000 habitantes (INE, 2002).

\section{TIPO DE CLIENTES}

Los clientes admitidos a este servicio de salud cubren todas las edades, siendo la gran mayoría adultos mayores. Generalmente estos 
clientes son pacientes crónicos, que han sufrido accidentes vasculares o son víctimas de cáncer o senilidad, con un promedio de edad deaproximadamente 76 años (Santiagos, 2002 información personal). Esto es confirmado por Joel (2001), quien indica que la atención domiciliaria frecuentemente es ofrecida al paciente debilitado con una condición crónica. Como se mencionó anteriormente, los clientes admitidos a este servicio domiciliario deben tener seguros de salud o alternativamente la capacidad de financiar los costos del servicio en forma privada.

\section{MODOSDE INGRESO}

Los clientes son referidos a este servicio de salud por el médico tratante o por consulta directa al servicio. Una vez ingresados al sistema, una enfermera visita al cliente en su domicilio y junto con el diagnóstico médico valora la condición de salud del paciente para luego iniciar el tratamiento indicado. Los clientes son admitidos siguiendo pautas de salud previamenteestablecidas. El nivel al que ingresan los clientes es categorizado deacuerdo al tratamiento y a la ayuda requerida para satisfacer sus necesidades básicas. Esasí como, en algunos casos, se puede requerir atención de enfermería continuada entre 12 o 24 horas diarias.

\section{NIVELES DE ATENCIÓN}

Como una forma de organizar la atención domiciliaria y categorizar el nivel de atención domiciliaria requerida, la agencia de salud aplica tres criterios:

- Nivel 1. Requiereatención de enfermería por 24 horas, el cual ofrece atención de auxiliares de enfermería durante las primeras horas. Ésta se traslada al hogar y queda permanentemente al cuidado del paciente, el cual es visitado diariamentepor la enfermera. Dependiendo dela condición del paciente, esta visita puede ser una o dos veces al día o con la frecuencia que sea necesaria. Duranteeste periodo el equipo desalud permanece en comunicación constante con la enfermera tratante.

- Nivel 2. También requiere atención de enfermería por 24 horas a cargo de un auxiliar de enfermería, pero solamente con visita de enfermera dos o tres veces a la semana.

- Nivel 3. Se refierea clientes que sólo requieren ser controlados una vez a la semana o una vez al mes por la enfermera.

Hay quedestacar quela gravedad delos clientes en nivel 1 y 2 frecuentemente requieren de equipos especiales que van desde un catre clínico, ropa de cama a un ventilador mecánico.

En orden de mantener el cuidado continuado, los datos obtenidos durante el ingreso y el cuidado posterior quedan registrados en una ficha clínica, que permanece en el domicilio del cliente. Solamente el personal de enfermería y el médico tratantetienen acceso a esta información.

\section{ROL DE LA FAMILIA}

A pesar que no es el objetivo de este artículo discutir el rol de la familia, es importante enfatizar que en el cuidado domiciliario la familia juega un rol esencial. Se sabequela participación de la familia en el cuidado del enfermo es fundamental, especialmente en el periodo de recuperación y convalescencia (Kellett, 1999). Las enfermeras que trabajan en el sistema (ADP) de atención domiciliaria tienen contacto directo y frecuente con los familiares del enfermo, especialmente con la persona responsable que generalmente es la esposa o la madre o la hija. En otras oportunidades puedeser el esposo, el padreo un hijo. En los casos de adultos mayores, éstos pue- 
den vivir solos, con sus hijos, con una dama de compañía o alternativamente pueden ser trasladados temporalmente al domicilio del familiar designado.

Laliteratura describeal gunos estudiosque han examinado las relaciones interpersonales entre enfermeras y miembros de una familia (Stiles, 1994; H upcey, 1998). Algunos de estos estudios describen los problemas y tensionesquefrecuentemente ocurren en los hospitales entre familiares y el personal de enfermería. Por ejemplo, en un estudio conducido por Hupcey (1999) en unidades de cuidado intensivo, se mostró que las relaciones interpersonales entre enfermeras y familiares y la participación de éstos en el cuidado del enfermo no era promovida. Además algunos miembros de la familia ocupaban su tiempo evaluando a la 'buena enfermera/o' y comparándola/o entreellas/os y pasando esta impresión a otros familiares. Por otro lado, las enfermeras consideraron queal gunos miembros de la familia mostraban falta de confianza en el cuidado deenfermería(Hupcey, 1998). Otros estudios han indicado quelas enfermeras evitan enfrentarse con los familiares porque no tienen suficiente tiempo (Bond, 1982). Una situación diferenteespresentada por Coffman (1997) quien encontró quelosfamiliaresapreciaban el tiempo que las enfermeras permanecían con el familiar enfermo y en el caso de Bridgman y Carr (1997) encontró quelas enfermeras que proveían cuidado en el hogar tenían una relación positiva con la familia y al mismo tiempo hacían participar a éstos en el cuidado del paciente. La situación de las enfermeras domiciliarias chilenas no es diferente. A pesar de que frecuentemente se ven enfrentadas por la angustia y frustración de los familiares que a menudo tienen expectativasirreales dela condición del cliente, en su gran mayoría estos problemas se solucionan con una comunicación efectiva. Es aquí donde la enfermera domiciliaria se relaciona directamente con la familia haciéndolos comprender la situación de salud y en la gran mayoría de los casos los invita a participar en el cuidado del paciente (Santiagos, comunicación personal, 2002).

\section{CARGA DE TRABAJO Y CUIDAD 0 CONTINUO}

El objetivo de este servicio (ADP) de salud además de prestar el cuidado a nivel del hogar es de ofrecer atención continua. Cada enfermera tiene que visitar aproximadamente seis pacientes (Fig. 1). Estos clientes pueden ser niveles 1 y 2 o 2 y 3 . El tiempo de atención de enfermería designada para cada visita domiciliaria es de dos horas. Si eventualmente este tiempo se extiende por una situación de urgencia 0 algún otro imprevisto, el servicio puede responder a estas emergencias reorganizando las visitas pendientes. Generalmente estos domicilios están distribuidos dentro de una área geográfica cercana. Las enfermeras tratantes deben proveer su propio vehículo, el recorrido diario es de aproximadamente60 $\mathrm{km}$. Ocasionalmente el cliente puede vivir fuera del área asignada a la enfermera, lo cual puede significar unos $20 \mathrm{~km}$ extra de recorrido. Las enfermeras son reembolsadas por el uso del combustibley la depreciación del vehículo. 
Figura 1. Carga diaria de trabajo.

\begin{tabular}{|c|c|c|}
\hline Cliente & Edad & Diagnóstico y tratamiento \\
\hline $\begin{array}{l}1 \\
M V\end{array}$ & 3 días & $\begin{array}{l}\text { Hiperbilirrubinemia } \\
\text { Fototerapia continua }\end{array}$ \\
\hline $\begin{array}{c}2 \\
G\end{array}$ & 85 & $\begin{array}{l}\text { Accidente vascular encefálico. } \\
\text { Alimentación por sonda nasoyeyunal, tratamiento endovenoso, sonda } \\
\text { Foley. } \\
\text { Kinesi oterapia. }\end{array}$ \\
\hline $\begin{array}{c}3 \\
C G\end{array}$ & 60 & $\begin{array}{l}\text { Cáncer pulmonar, tratamiento oncológico endovenoso, hidratación } \\
\text { parenteral, curación }\end{array}$ \\
\hline $\begin{array}{c}4 \\
\mathrm{RP}\end{array}$ & 65 & $\begin{array}{l}\text { Tromboflebitis } \\
\text { Tratamiento con solución heparina por bomba de infusión }\end{array}$ \\
\hline $\begin{array}{c}5 \\
J P\end{array}$ & 7 & $\begin{array}{l}\text { Amigdalitis pultácea } \\
\text { Tratamiento endovenoso cada } 8 \text { horas sólo por enfermera }\end{array}$ \\
\hline $\begin{array}{c}6 \\
M P\end{array}$ & 78 & $\begin{array}{l}\text { Accidente vascular encefálico. } \\
\text { Tratamiento con solución heparina por bomba de infusión. }\end{array}$ \\
\hline
\end{tabular}

Por razones obvias de confidencialidad y privacidad, el nombre de los pacientes se ha reemplazado por iniciales.

\section{COSTOS}

Loscostos dela atención prestadavan deacuerdo a la condición del clientey la duración del servicio prestado. Deacuerdo con Dukey Street (2003), la atención domiciliaria generalmente disminuye el costo de la atención otorgada y en su gran mayoría los gastos son cubiertos por los seguros de salud. La atención domiciliaria no requiere de la maquinaria administrativa usada en el hospital. Joel (2001) informa que la atención domiciliaria redujo los costos de ciertas condiciones médicas como el cuidado de recién nacidos de bajo peso, de pacientes que requerían ventilación mecáni$\mathrm{ca}$, en niños con tratamientos de quimioterapia, pacientes con tratamientos endovenosos y con insuficiencia cardiaca (National Association for HomeCare, cita en Joel, 2001). En el caso delos pacientes chilenos, los gastos son cubiertos por el tipo de seguro de salud que tenga el beneficiario. En Chile estos seguros son conocidos con el nombre de Isapre (Instituto de Salud Previsional).

\section{ALGUNAS DIFICULTADES}

El trabajo de la enfermera/o domiciliaria en una ciudad como Santiago deChile, se duplica debido a la congestión vehicular, lo quedificulta y retrasa el desplazamiento entre los diferentes domicilios. Como se menciono anteriormente, este servicio ofrece atención de la enfermería a través de 24 horas, lo que significa que las enfermeras visitan a sus pacientes a diferentes horas del día y la noche. Cabe destacar que especialmente en las noches las dificultades aumentan debido a la mala señalización y numeración delos domicilios como también los riesgos de posible asaltos. Es de esperar quelas dificultades con queseenfrentan las enfermeras domiciliarias no tengan mayor repercusión en el nivel de satisfacción profesional, ya queestudios conducidos en los Estados Unidos deNorteAmérica indican que la atención domiciliaria está en crisis debido a la escasez de enfermeras, como asimismo a un descontento con sus trabajos; esto es alarmante, especial mentecuando la necesidad de 
atención domiciliaria va aumentado (Flyn \& Deatrick, 2003).

\section{ALGUNAS PREGUNTAS}

No obstante las dificultades mencionadas anteriormente, las enfermeras domiciliarias están desarrollando una labor que solamente es conocida por aquellos queseben efician con su cuidado, labor que hasta el momento es desconocida por la gran mayoría dela población. De todas maneras, vale la pena preguntarse, ¿cuál es el futuro de la enfermería domiciliaria en Chile? Ciertamente, aquel dependerá, en gran medida, de la experiencia adquirida por estas profesionales a través de los años y quela infraestructura dela institución sea capaz de mantener un servicio de calidad. Además, dependerá del alza de los costos de hospitalización y las condiciones económicas de la población en general. Cabe esperar que la atención domiciliaria adquiera prioridad como sistema de salud, pues hasta la fecha ésta no se ha desarrollado en su totalidad. Las metas planteadas por la enfermería domiciliaria para los próximos años no podrán lograrse sin el apoyo de la familia y sus beneficiarios. Es más, la familia está desempeñando un rol fundamental al elegir un sistema de atención quefavoreceal paciente, en su propio hogar. La tendencia que día a día se está promoviendo en el ámbito de la salud es el cuidado en el hogar, una realidad tangible que está al alcance de aquellos que poseen un sistema previsional de salud. A pesar de los aspectos positivos que estemodelo deatención ofrece, hay ciertas áreas que necesitan ser exploradas. Por ejemplo, ¿cuánto se sabe si el cuidado en el hogar está causando problemas de tipo físico o emocional en la familia? o ¿es aceptable la pérdida de la privacidad e intimidad de aquélla cuando el hogar está abierto al escrutinio de los profesionales de la salud? (Cooper, cita en Duke \& Street, 2003). De todas maneras, cabe felicitar a las enfermeras que desarrollan este tipo de cuidado, al mismo tiempo se espera que las investigaciones basadas en la evidencia práctica de muestren si la atención domiciliaria ha sido de calidad.

\section{UN MODELO DE ATENCIÓN DOMICILIARIA}

Existe una clara indicación en el ambiente de la salud de la necesidad de buscar nuevas oportunidades para entregar estos servicios a la comunidad. La atención de salud a nivel del sistema hospitalario ha sido predominanteen las áreas de cuidado agudo, además de la salud mental, cuidado del anciano y el niño (Gursanski, Harvey \& Kennedy, 2003). Muchos de estos cambios se deben a que las personas con problemas agudos como asimismo aquellos con enfermedades crónicas pueden tener ayuda en sushogares y continuar viviendo independientemente. Otro de los factores quehan facilitado este movimiento hasido la demanda de los pacientes con relación al derecho de poder elegir el sistema de salud que más les acomode. Los cambios de las políticas de salud en los países desarrollados, han sido de un sistema que ha enfatizado la responsabilidad de la familia en el cuidado de los enfermos. Consecuentemente, los principios que guían las pautas del modelo de atención domiciliaria son básicamente los siguientes:

1. Un servicio diseñado a prestar atención individualizada.

2. Participación de la familia en el cuidado del enfermo.

3. Oportunidad de elegir el servicio de salud disponible.

4. Ofrecer un servicio de calidad a un costo adecuado.

5. Mantener una continuidad en el cuidado (Gursanski, H arvey \& Kennedy, 2003).

Cabe destacar que el éxito del modelo de atención domiciliaria depende de varios factores. Por ejemplo, la calidad del servicio do- 
miciliario está basado fundamentalmente en la filosofía que promueve la agencia de lo que significa el cuidado domiciliario. Además del entrenamiento continuo del personal queasegure la calidad del cuidado, en una revisión sistemática delaatención prestaday en la estabilidad del equipo desalud (DementiaServices, 1997).

\section{CONCLUSIÓN}

Este artículo ha tratado de ilustrar en forma breve un modelo de atención domiciliaria prestada a un sector de la población de Santiago de Chile. A pesar que la información es anecdótica, ésta representael comienzo deuna tarea urgente y más profunda, la cual es conducir estudios sistemáticos. El próximo paso quelas enfermeras deben iniciar es promover la investigación en el área de la atención domiciliaria. Esta posición es justificada por otros autores (Duke \& Street, 2003), quienes indican que el cuidado en el hogar se está expandiendo a pesar de no haber suficiente investigación que justifique la necesidad de continuar ofreciendo enfermería domiciliaria como una alternativa de atención deseable. Además, la única forma de demostrar que este modelo deatención es efectivo es demostrando, tanto en el ámbito nacional como internacional, el impacto que la atención de enfermería domiciliaria está produciendo en la comunidad.

\section{REFERENCIAS}

Bond, S. (1982). Communicating with families of cancer patients: Thenurses. N ursingTimes, 6(16), 1027-1029.

Bridgman, H \& \& Carr, E. (1997). The provision of family-focused palliative care in hospital using the Delphi technique. NT Research, 2(6).

Bryas, A.N . (2004). Examining health visiting expertise: Combining simulation, interview and observation. Journal of Advanced N ursing. 47(6), 623-630.

Coffman, S. (1997). Home-care nurses as strangers in the family. Western Journal of N ursing Research, 19(1), 82-95.
Dementia Services D evelopment Centre (1997). Case management. In enabling care practices-from philosophy to evaluation (DSDC). Hammond Care Group. http://www.hammond.com.au/resources/ providers details. php?rid=7. Retrieved 26/02/04.

Duke, M \& \& Street, A. (2003). Theímpetus for the development of hospital in the home (HITH) programs: A literature review. Contemporary Nurse, 14(3), 227-239.

Flynn, L. \& Deatrick, J.A. (2003). Home care nurses' descriptions of important agency attributes. Journal of N ursing Scholarship, 35(4), 385-390.

Gursanski, D., Harvey, J. \& Kennedy, R. (2003). Case management. Policy, practice, and profesional business. Crows Nest, N SW: Allen \& Unwin.

Joel, L.A. (2001). M oving the care. From hospital to home, from nurses to whom? In J. M c. Dochterman $\&$ H. K. Grace. Current issues in nursing (6th ed.) (pp. 142-149). St Louis: M osby.

Hughes, I. C., Hodgson, N. A., M uller, P., Robinson, L. A. \& M cCorkle, R. (2000). Information needs of elderly postsurgical cancer patients during thetransition from hospital to home. Journal of Nursing Scholarship, 32(1), 25-30.

Hupcey, J.E. (1998). Establishing the nurse-family relationship in the intensive care unit. Western Journal of N ursing Research, 20(2), 180-194.

Hupcey, J.E. (1999). Looking out for the patient and ourselves - the process of family integration into ICU. Journal of Clinical N ursing, 8(3), 253-262.

Instituto Nacional de Estadística. Información censo 2002. www.ine.cl/cd 20002/index.php Datos obtenidos el 20 de abril de 2004.

Kearney, M. H., York, R. \& D eatrick, J. A. (2000). Effects of home visits to vulnerable young families. Journal of Nursing Scholarship, 32(4), 369-376.

Kellett, U. (1999). Meaning in caring: Reconceptualizing the nurse-family carer relationship in community practice. Journal of Advanced Nursing, 29(3), 697-703.

Santiagos, A . (2002) . Comunicación personal . Febrero, 2002.

Schneider, H. (2001). Chileans in Jupp, J. (Ed). The Australian people. An encyclopedia of the nation, its peopleand their origins. Centrefor immigration and multicultural studiesAustralian National University: Cambridge University Press. pp.195-197.

Stiles, M .K. (1994). The shining stranger: Application of the phenomenological method in the investigation of the nurse-family spiritual relationship. Cancer N ursing, 17(1), 18-26.

Wright, L.M . \& Leahey, M . (1994). N urses and families: A guideto family assessment and intervention (2nd ed.). Philadelphia: F.A. Davis. 